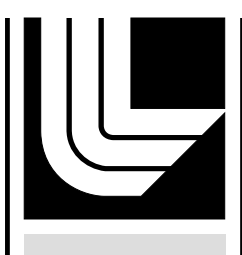

LAW RENCE LIVERMORE N A TIO N A L LABORATORY

\title{
Multimode-diode-pumped gas (alkali-vapor) laser
}

R. H. Page, R. J. Beach, V. K. Kanz

August 29, 2005

Optics Letters 
This document was prepared as an account of work sponsored by an agency of the United States Government. Neither the United States Government nor the University of California nor any of their employees, makes any warranty, express or implied, or assumes any legal liability or responsibility for the accuracy, completeness, or usefulness of any information, apparatus, product, or process disclosed, or represents that its use would not infringe privately owned rights. Reference herein to any specific commercial product, process, or service by trade name, trademark, manufacturer, or otherwise, does not necessarily constitute or imply its endorsement, recommendation, or favoring by the United States Government or the University of California. The views and opinions of authors expressed herein do not necessarily state or reflect those of the United States Government or the University of California, and shall not be used for advertising or product endorsement purposes. 


\section{Multimode-diode-pumped gas (alkali-vapor) laser}

Ralph H. Page, Raymond J. Beach, and V. Keith Kanz

Lawrence Livermore National Laboratory

7000 East Ave., Livermore CA 94551-0808

We report the first demonstration of a multimode-diode-pumped gas laser $-\mathrm{Rb}$ vapor operating on the $795 \mathrm{~nm}$ resonance transition. Peak output of $\sim 1$ Watt was obtained using a volume-Bragg-grating stabilized pump diode array. The laser's output radiance exceeded the pump radiance by a factor greater than 2000 . Power scaling (by pumping with larger diode arrays) is therefore possible.

OCIS codes: $140.3480 \quad 140.1340 \quad 140.2010 \quad 020.2070$

Optically-pumped alkali-vapor lasers date back to 1962 or so. [1] The $389 \mathrm{~nm}$ output from a He lamp fortuitously coincides with a $6 \mathrm{~s}-8 \mathrm{p}$ transition in Cs, enabling four-level-laser operation on the $8 p-8 s$ and $8 p-6 d$ transitions at 7.18 and $3.20 \mu \mathrm{m}$, respectively. Although scientifically very interesting, such schemes produce inherentlyinefficient lasers because of the order-of-magnitude difference in pump and laser wavelengths.

On the other hand, resonance-transition alkali-vapor lasers are noteworthy for their potentially-high efficiency: the energies of the $\mathrm{D}_{1}$ (pump) and $\mathrm{D}_{2}$ (laser) transitions 
differ at most by few percent. Furthermore, the sparseness of the energy levels and resonances eliminates excited-state absorption (ESA.) So, alkali vapors are attractive candidates for further development. Since the resonance transitions of several (especially $\mathrm{K}, \mathrm{Rb}$, and $\mathrm{Cs}$ ) are in the near infrared, pumping with diode lasers is a primary goal.

Use of the alkali $D_{1}$ and $D_{2}$ transitions presents special problems. [2] Population in the ${ }^{2} \mathrm{P}_{3 / 2}$ level does not radiatively relax into the ${ }^{2} \mathrm{P}_{1 / 2}$ level, so a molecular collision partner (ethane [3]) is used to accelerate thermal equilibration and prevent "bottlenecking." The sharpness of the atomic lines at typical cell temperatures (100 - 200 ${ }^{\circ} \mathrm{C}$ ) makes broadband pumping impractical unless pressure-broadening (with $\mathrm{He}$ ) is employed. And, the three-level nature of the scheme mandates pumping on the scale of the broadband saturation intensity (per unit bandwidth) $\mathrm{I}_{\mathrm{sat}}=4 \pi \mathrm{hv} / \lambda^{2}$ in order to achieve net gain. When converted to wavelength units and evaluated at $780 \mathrm{~nm}\left(\mathrm{Rb} \mathrm{D}_{2},\right) \mathrm{I}_{\mathrm{sat}}$ corresponds to $260 \mathrm{~W} / \mathrm{cm}^{2} \cdot \mathrm{nm}$. Because the optical path density NoL is typically several nepers, even higher brightness is required for bleaching the transition, and laser threshold is achieved at several times $\mathrm{I}_{\text {sat }}$, or a few $\mathrm{kW} / \mathrm{cm}^{2} \cdot \mathrm{nm}$.

Birefringent-filter-tuned Ti:sapphire lasers, whose narrow linewidths and excellent mode quality make them extremely bright pump sources, were used in the first demonstrations of resonance-transition alkali-vapor lasers. [2] Subsequently, a diode- 
pumped Cs laser was reported. [4] Since its pump source was a tunable master-oscillator / power-amplifier (MOPA) system, whose beam quality and brightness are also very high, it remained an open challenge to demonstrate laser operation with a "low-coherence" pump source such as a diode array.

In both experiments, high slope efficiencies were achieved — several tens of percent. Rate-equation modeling with no adjustable parameters [5] well reproduced extensive data for a Ti:sapphire-pumped Cs-vapor laser, giving confidence that the essential physical principles were well-understood. Adjustability of the pump linewidth and pressure-broadened atomic linewidths (as model parameters) enabled extrapolation to the "power-scaled" situation employing extremely large pump powers (tens of kW) delivered by highly-multimode pump arrays of linewidth $\sim 2 \mathrm{~nm}$. Using an unstableresonator, zig-zag geometry with high "mode fill," optical-to-optical efficiencies of 70\% were predicted to be realizable. Face cooling and a negative thermo-optic coefficient $d n / d T$ were estimated to make the "thermal management" problem tractable.

A schematic diagram of the setup used in the present experiment is shown in Figure 1. At its heart is a heated cell whose interior windows are formed from sapphire mini-Conflat flanges. A single-layer $\mathrm{MgF}_{2} \mathrm{AR}$ coating was applied to the windows, whose gap is $2.25 \mathrm{~mm}$, roughly the depth of focus of the pump-diode light. Pump light enters the nearly-confocal cavity via a "holey mirror" produced by drilling a $2.0 \mathrm{~mm}$ hole at $45^{\circ}$ into a silver-coated piece of polished 304 stainless steel. The $0.4 \mathrm{~mm}$-diameter output of a fiber-coupled, volume-Bragg-grating-stabilized diode array is imaged into the 
cell with a telescope of magnification 1.5 to produce a $0.6 \mathrm{~mm}$ pump spot. This reduces the numerical aperture from 0.22 (at the fiber output) to $\sim 0.15$ (in the cell.) The "mode fill" (geometric overlap) $\omega_{1}{ }^{2} / \omega_{\mathrm{p}}{ }^{2}$ of resonated and pump spot sizes is 0.28 in this arrangement. With pristine cell windows, roughly $90 \%$ of the pump light was transmitted into the alkali-vapor region. For detecting the laser output, a $795 \mathrm{~nm}$ interference filter was followed by a calorimeter and/or Si photodiode-based power meter.

The 2-piece cell included a valve for connection to a gas manifold. It was baked under vacuum, then loaded with Rb metal in an Ar-purged glove bag. Upon reassembly, it was pumped at room temperature, then filled with 90 torr ethane, and with He for a total pressure of $2 \mathrm{~atm}$. Because a drop of $\mathrm{Rb}$ stuck to one of the windows during loading, we lost the ability to prevent condensation of $\mathrm{Rb}$ on the optical surfaces. The cell was sandwiched in a heater assembly based on a hollowed-out copper block. Air gaps and exterior AR-coated windows facilitated maintenance at elevated temperature (ostensibly preventing alkali-metal condensation) of the interior cell windows. An XYZ stage was used for positioning the cell accurately with respect to the pump focus.

The "L-I curve" for the Spectra-Physics pump-diode array is shown in Figure 2(a). To simplify achieving the triple coincidence between the resonant wavelengths of the semiconductor gain medium, the volume Bragg grating, and the $\mathrm{Rb} \mathrm{D}_{2}$ line, the diode was run at a temperature near $25^{\circ} \mathrm{C}$ and pulsed at $100 \mathrm{~Hz}, 500 \mu \mathrm{sec}$ (i.e. $5 \%$ duty cycle.) In this mode, the diode output spectrum consisted of a narrow feature $(\sim 0.3 \mathrm{~nm}$ wide; the "resonant fraction") at $780.25 \mathrm{~nm}$ and a $\sim 3 \mathrm{~nm}$-wide hump near $777 \mathrm{~nm}$ (see Figure 2 
(b).) At the maximum drive current of $40 \mathrm{~A}$, the resonant drive power was $14 \mathrm{Watt}$. Under these conditions, we estimated the peak power spectral density at $45 \mathrm{~W} / \mathrm{nm}$, corresponding to a peak brightness (inside the cell) near $14 \mathrm{~kW} / \mathrm{cm}^{2} \cdot \mathrm{nm}$.

With the cell at $190{ }^{\circ} \mathrm{C}$, it was possible (see Fig. 3) to pump the laser at $\sim 3$ times threshold. Insensitivity of the pump threshold to output coupling is a consequence of operating with a large column density $\mathrm{N} \sigma \mathrm{L} \sim 38$. Improvement of slope efficiency with increased output coupling is a consequence of significant intracavity loss. Figures 3(b) and 3(c) show respectively the pump threshold and maximum output power as a function of cell temperature, using the $50 \%$ output coupler. At the lowest operating temperature $\left(135^{\circ} \mathrm{C}\right.$,) the column density is barely enough to produce gain in excess of the cavity loss. On the other hand (at $200{ }^{\circ} \mathrm{C}$, the limit of our temperature controller, and roughly a factor of 20 greater column density,) the threshold rises because more power is needed to bleach the ground-state absorption. The maximum output power ( $\sim 1$ Watt $)$ occurs at a cell temperature $\sim 20^{\circ} \mathrm{C}$ higher than the minimum threshold because the pump light is absorbed more completely.

The best slope efficiency achieved with this setup was $\sim 10 \%$. It was found that during laser operation, cell window transmission degraded where the pump light entered. Cold-cell measurements revealed a transmittance of $\sim 0.7$ (leading to an overall pump transport efficiency of only $\sim 0.6$.) Coupled with the low mode fill of $\sim 0.28$, the observed efficiency is in rough agreement with predictions based on the model of Ref. [5.] Because the intracavity loss etc. varied with time, global modeling of the dataset in Fig. 3 
is not realistic. It remains to be seen whether keeping alkali metal off the interior window surfaces (or a different choice of window or coating material) will prevent the buildup of performance-degrading deposits.

The "radiance enhancement" obtained with the diode-pumped alkali-vapor laser (DPAL) can be estimated by considering the relative spot sizes, divergences, and powers of the pump and laser beams. Following the treatment of Siegman, [6] we can associate the multimode pump beam's $0.2 \mathrm{~mm}$ spot radius and numerical aperture of 0.22 with an analogous Gaussian beam that is 177 "times diffraction limited." With a peak power of 14 Watt, the resultant brightness is $\mathrm{P}_{\text {pump }} / \mathrm{A}_{\text {pump }} \Omega_{\text {pump }}=7.3 \times 10^{-4} \mathrm{~W} / \mu \mathrm{m}^{2}$-sterad. Assuming a diffraction-limited beam and calculating the $1 /$ e effective spot size-cone angle containing $86 \%$ of the output power, the analogous value for the 1 -Watt DPAL is $\mathrm{P}_{\text {laser }} / \lambda^{2}=1.58 \mathrm{~W} / \mu \mathrm{m}^{2}$-sterad; thus, the brightness was increased a factor of $\left(\mathrm{P}_{\text {laser }} / \mathrm{P}_{\text {pump }}\right)\left(\mathrm{A}_{\text {pump }} \Omega_{\text {pump }} / \lambda^{2}\right)=2160$. Performing the analogous calculation for the $\mathrm{Rb}$ resonance-line laser of Krupke et al [2] (29 $\mathrm{mW}$ output with $178 \mathrm{~mW}$ pump power) gives a radiance multiplication of 0.16 . For the $895-\mathrm{nm}$ Cs resonance-line laser of Ehrenreich et al [4,] which produced $130 \mathrm{~mW}$ with $370 \mathrm{~mW}$ pump power, the radiance multiplication is 0.32 . Thus, the present experiment represents important progress in the use of alkalivapor lasers to convert incoherent diode pump light into highly-coherent output light.

Having demonstrated DPAL operation with multimode, modestly-coherent pump diodes and having achieved an overall brightness multiplication, it can credibly be 
alleged that increasing the output power of such a laser could be accomplished by adding more pump-diode arrays. Cleverly multiplexing many diode arrays, and confining their combined output in a multimode guided structure, can serve to maintain the pump flux above the $\mathrm{I}_{\text {sat }}$ value needed to invert the three-level alkali-vapor system. Thus we look forward to continued development of DPALs, at ever-higher power.

We are grateful to W. F. Krupke for numerous technical discussions, and to Alan Petersen, Keeley Hurley, Jim Harrison, Georg Treusch, John Gloyd, and Ed Wolak of Spectra-Physics for wavelength-stabilized diode arrays. We also gratefully acknowledge the support of the LLNL LDRD program. This work was supported by the U.S. Department of Energy under Contract W-7405-ENG-48 and the High Energy Laser Joint Technology Office (HEL JTO).

\section{References}

[1] P. Rabinowitz, S. Jacobs, and G. Gould, "Continuous optically pumped Cs laser," Appl. Optics 1, 513 (1962.)

[2] W. F. Krupke, R. J. Beach, V. K. Kanz, and S. A. Payne, "Resonance transition 795nm rubidium laser,” Opt. Lett. 28, 2336 (2003.)

[3] Z. Konefal, "Observation of collision-induced processes in rubidium-ethane vapour," Opt. Commun. 164, 95 (1999.) 
[4] T. Ehrenreich, B. Zhdanov, T. Takekoshi, S. P. Phipps, and R. Knize, "Diode pumped caesium laser," Electronics Lett. 41, 415 (2005.)

[5] Raymond J. Beach, William F. Krupke, V. Keith Kanz, Stephen A. Payne, Mark A. Dubinskii, and Larry D. Merkle, "End-pumped continuous-wave alkali vapor lasers: experiment, model, and power scaling," Beach J. Opt. Soc. Am. B 21, 2151 (2004.)

[6] Anthony E. Siegman, Lasers, pp. 672, 696 (University Science Books, Mill Valley, CA, 1986.) 


\section{Figure Captions}

Figure 1. A "holey mirror" directs pump light from a fiber-coupled, volume-Bragggrating stabilized pump diode array into the center of a short (2.25 mm-gap) cell positioned near the center of a nearly-confocal resonator.

Figure 2. (a) "L-I curve" for the pump-diode array. Part of its output was not locked by the volume Bragg grating. (b) Diode-array spectrum obtained at 40 A drive current, showing the $780.25 \mathrm{~nm}$ peak resonant with the $\mathrm{Rb}$ pump transition.

Figure 3. (a) Slope plots obtained with four different output couplers, with the cell at $190{ }^{\circ} \mathrm{C}$. (b) Threshold drive power vs cell temperature, with the $50 \%$ output coupler. (c) Peak output power vs cell temperature, using the $50 \%$ output coupler. 


\section{diode array $\rightarrow$}

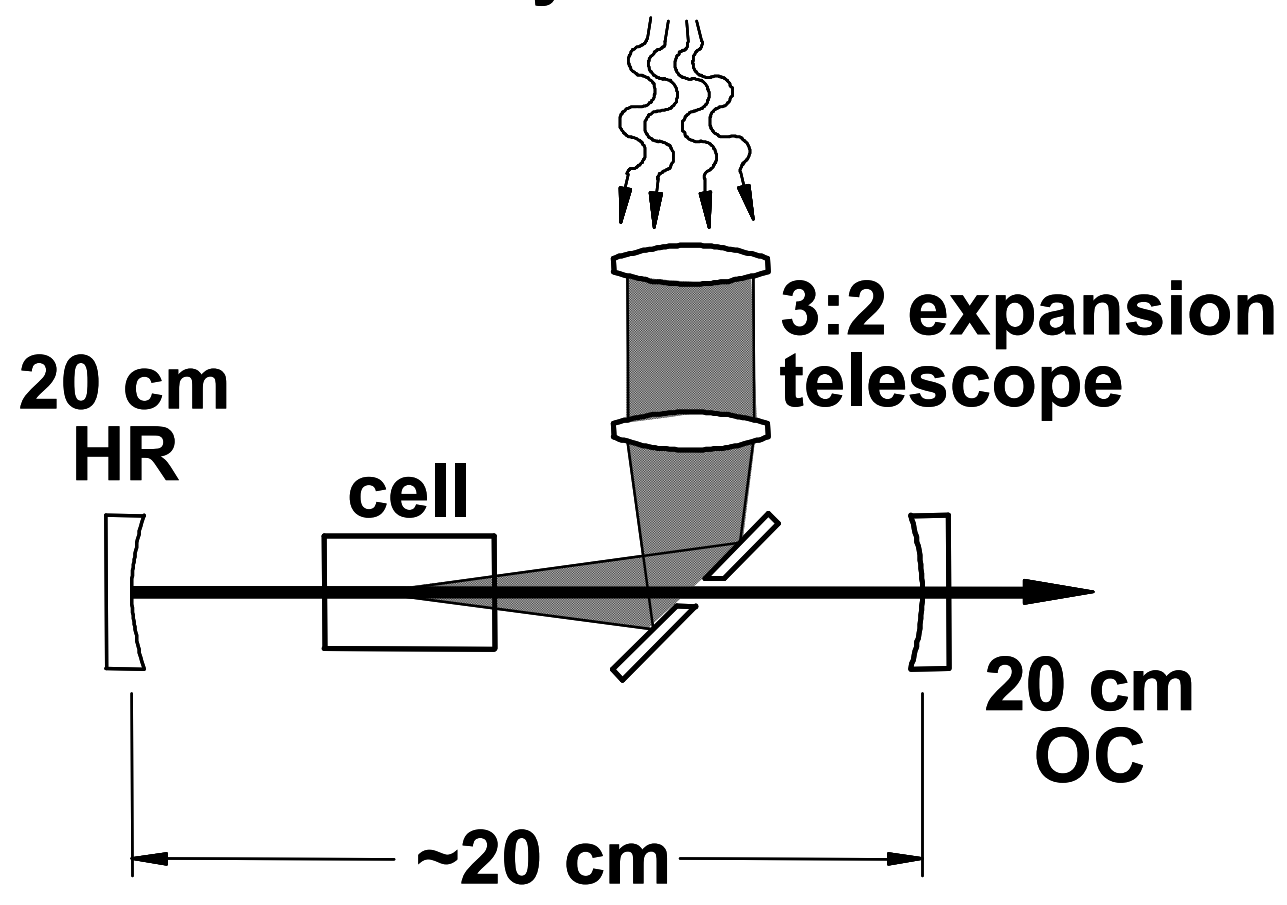

Figure 1. A "holey mirror" directs pump light from a fiber-coupled, volume-Bragggrating stabilized pump diode array into the center of a short (2.25 mm-gap) cell positioned near the center of a nearly-confocal resonator. 

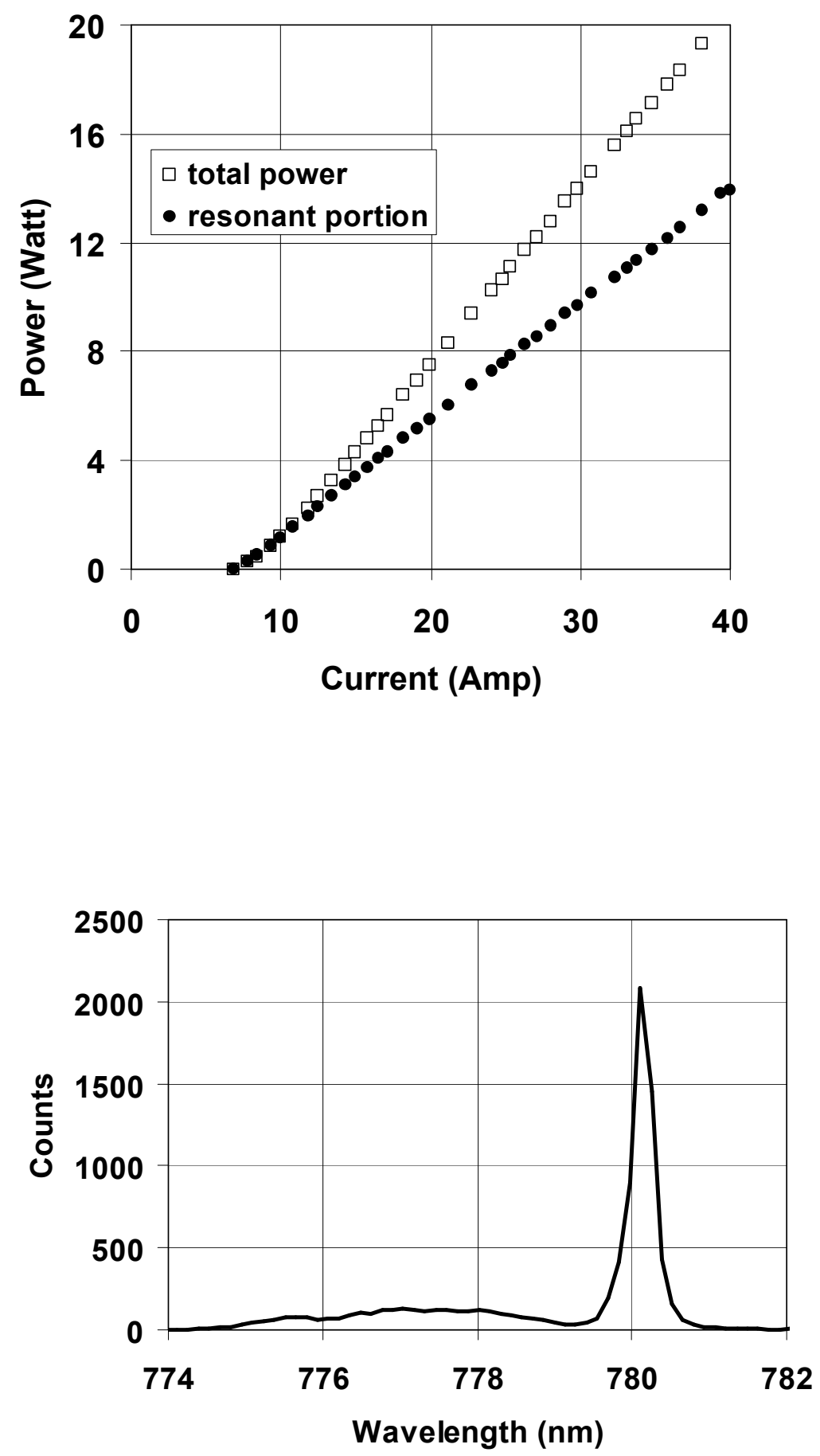

Figure 2 (a). "L-I curve" for the pump-diode array. Part of its output was not locked by the volume Bragg grating. (b) Diode-array spectrum obtained at 40 A drive current, showing the $780.25 \mathrm{~nm}$ peak resonant with the $\mathrm{Rb}$ pump transition. 

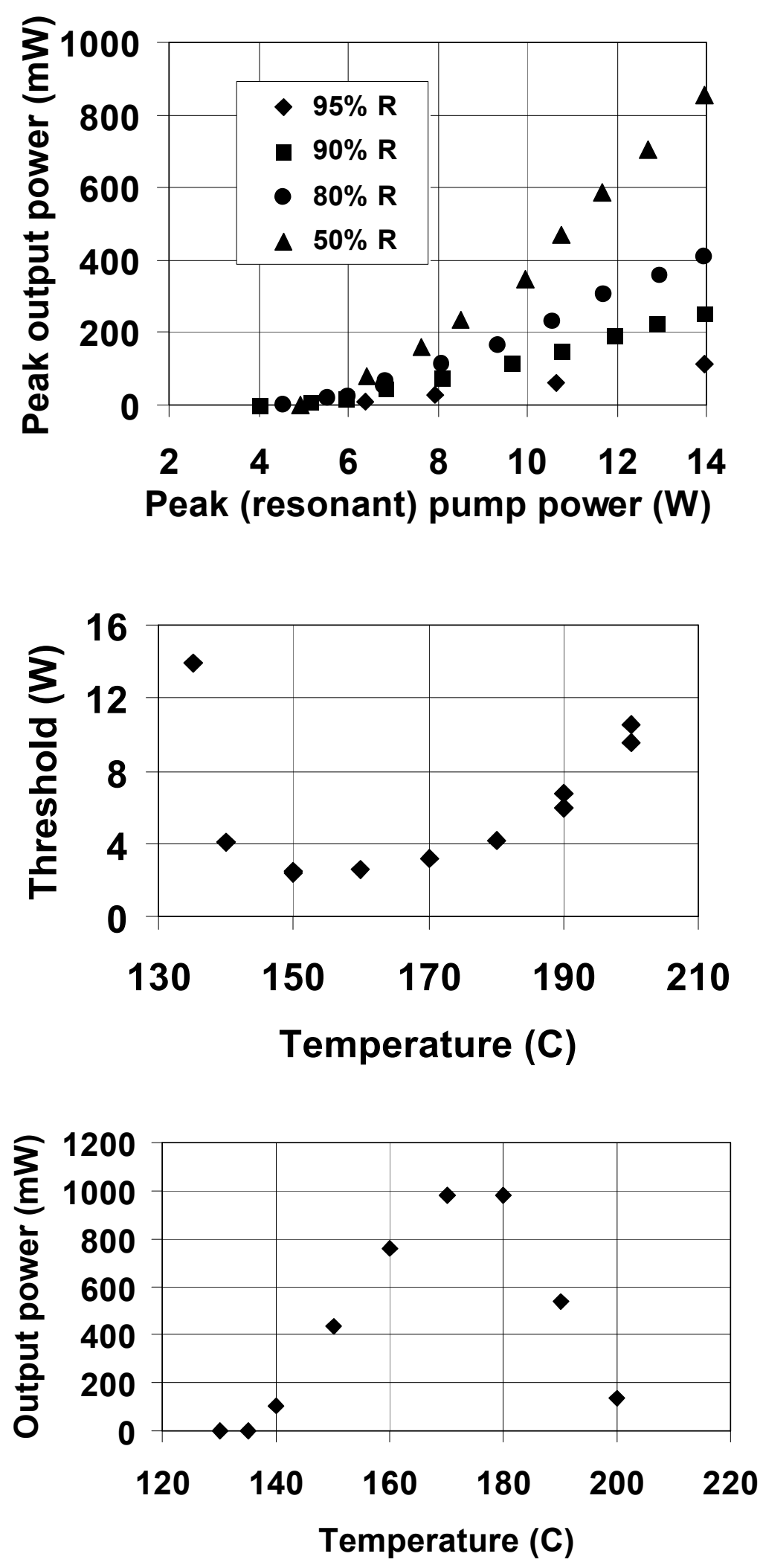

Figure 3. (a) Slope plots obtained with four different output couplers, with the cell at $190{ }^{\circ} \mathrm{C}$. (b) Threshold drive power vs cell temperature, with the $50 \%$ output coupler. (c) Peak output power vs cell temperature, using the $50 \%$ output coupler. 